\title{
Attosecond Synchronization of High-Order Harmonics from Midinfrared Drivers
}

\author{
G. Doumy, * J. Wheeler, C. Roedig, R. Chirla, P. Agostini, and L. F. DiMauro \\ Department of Physics, The Ohio State University, Columbus, Ohio 43210, USA
}

(Received 26 November 2008; published 6 March 2009)

\begin{abstract}
The group delay dispersion, also known as the attochirp, of high-order harmonics generated in gases has been identified as the main intrinsic limitation to the duration of Fourier-synthesized attosecond pulses. Theory implies that the attochirp, which is inversely proportional to the laser wavelength, can be decreased at longer wavelength. Here we report the first measurement of the wavelength dependence of the attochirp using an all-optical, in situ method [N. Dudovich et al., Nature Phys. 2, 781 (2006)]. We show that a $2 \mu \mathrm{m}$ driving wavelength reduces the attochirp with respect to $0.8 \mu \mathrm{m}$ at comparable intensities.
\end{abstract}

Attosecond science, or attophysics, is the study of electron dynamics at time-scales approaching the atomic unit of time ( 1 a.u. $\approx 24$ as) [1]. Currently, the production of attosecond pulses and the development of attophysics are based on high harmonic generation in gases, a nonperturbative, highly nonlinear process. Using this method, light pulses close to 100 as have been demonstrated either as an attosecond pulse train (APT) with a repetition period of half the fundamental cycle [2] or as a single pulse [3,4]. So far the carrier frequency of attosecond pulses has been limited to the extreme ultraviolet (XUV) to soft-x-ray domain $(30-80 \mathrm{eV})$ but significantly more applications will be enabled if shorter bursts of more energetic $\mathrm{x}$ rays can be created, particularly time resolved studies of corelevel and multielectron dynamics [5]. In order to evaluate the physical limitations it is important to understand the role of the driving field characteristics. The recent development of intense midinfrared femtosecond pulses with wavelength 5 times that of the commonly used titanium: sapphire laser system has opened new routes to extreme harmonic generation and attophysics [6,7]. In this report, a measurement of the driving wavelength dependence of the group delay dispersion or attochirp, a property crucial for attosecond pulse synthesis, is described.

High harmonic generation is a quantum mechanical process involving the absorption of a large number of photons, for which the usual nonlinear optics treatment breaks down. In a more effective description based on classical trajectories, a field-driven electron freed by intense field ionization is accelerated for approximately half an optical cycle, and then can recombine to emit an attosecond burst of light $[8,9]$. This process occurs twice every optical cycle, which in the frequency domain corresponds to a comb of odd-order harmonics of the fundamental driving field. The corresponding quantum mechanical treatment is based on the strong field approximation [10] in which the time-dependent one-electron dipole is calculated by solving the Schrödinger equation neglecting the influence of the Coulomb potential on the motion of the free electron wave packet. In this approximation the main contribution to the dipole comes from the quantum paths whose quasiclassical action is stationary and therefore follow the aforementioned classical trajectories. Consequently, classical mechanics is an excellent approximation to describe the motion of the wave packet in the continuum. In particular, the semiclassical model can determine the maximum harmonic energy and predict a dispersion of the recombination times, which corresponds to a spectral group delay dispersion (GDD) of the emitted harmonics (the attochirp). In general, the limiting effects of GDD on ultrashort pulse formation are well known [11]. In attosecond generation, the attochirp translates into consecutive harmonic orders not being emitted simultaneously [see Fig. 1(a)] and the ordering of the frequencies defines the sign of the attochirp. As a consequence, there exists an optimal bandwidth for the production of the shortest attosecond bursts, beyond which the pulse broadens as dispersion dominates $[2,12]$. This is an intrinsic limitation related to the microscopic process which can be partially compensated by propagating the pulses in a suitably dispersive medium $[2,13,14]$.

The dependence of the attochirp on the laser parameters can also be understood by using the semiclassical model. In this approximation, the frequency of the emitted harmonic is determined by the energy of the returning electron at recombination. This kinetic energy is uniquely defined by the phase of the fundamental field at which the electron is promoted in the continuum. Simple Newtonian calculations then show that for each optical cycle, a given harmonic energy can be reached by two different trajectories, shown in Fig. 1, that have been dubbed short and long (see [15], for instance). Another consequence is that the upper limit for the harmonic energy is given by $I_{p}+3.17 U_{p}$, where $I_{p}$ is the ionization potential, and $U_{p} \propto I \lambda^{2}$ is the ponderomotive potential for a fundamental intensity $I$ and wavelength $\lambda$.

In the representation of Fig. 1(b), the attochirp is given by the derivative of the curve of emission times as a 


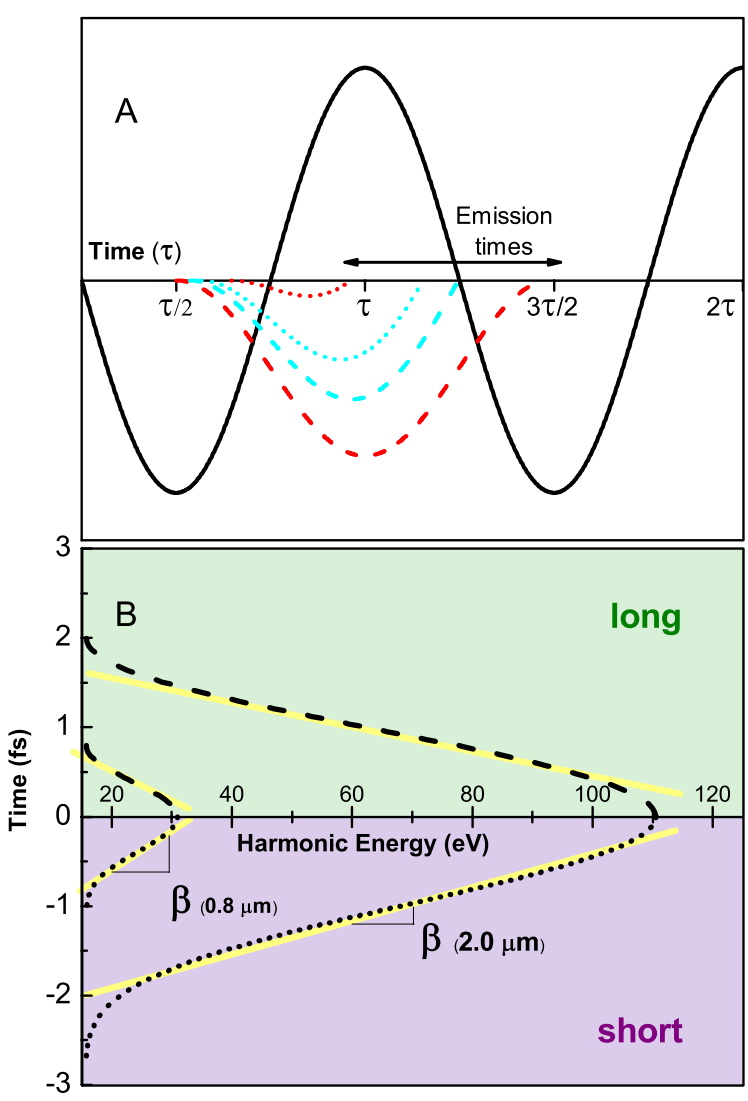

FIG. 1 (color online). (a) Representation of the short and long trajectories predicted by the semiclassical model. For a given phase of the fundamental field, tunnel ionized electrons have distinct trajectories. A selection is presented here, illustrating the different emission times of the harmonics. For each final energy, two different trajectories are possible (shown as the same color here), defining the two classes of trajectories (dots for short, dashes for long). The red (or dark gray) and blue (or gray) colors are used to illustrate high and low frequency harmonics, respectively. (b) The short trajectories are emitted first, and exhibit a positive chirp, $\beta$ [red (or dark gray) before blue (or gray) in (a)], which gets represented here as the slope of the graph. Increasing the wavelength [e.g., from 0.8 to $2 \mu \mathrm{m}$ ], extends the cutoff energy and the laser period, but lowers the attochirp as evidenced here by the decrease in slope.

function of harmonic energy. It is easy to see that for a given class of trajectories the attochirp is almost constant (i.e., nearly perfect quadratic spectral phase). It also follows that the attochirp is proportional to the ratio of the fundamental laser cycle period to the harmonic cutoff energy. Since the cutoff energy is proportional to the laser intensity, the obvious first way to reduce the attochirp is to increase the peak intensity [2]. However, this is limited to a maximum intensity corresponding to the depletion of the ground state (saturation).

A more subtle way to reduce the attochirp consists in exploiting the wavelength scaling. Since the laser period is proportional to $\lambda$ and the cutoff energy to $\lambda^{2}$, their ratio, and thus the attochirp should scale as $\lambda^{-1}$. As was argued in [7], increasing the fundamental wavelength at constant intensity avoids the problems related to ionization. Coupled with strong enhancement in the cutoff energy, this route would allow a better path towards shorter attosecond pulses centered at higher photon energies.

Here we report the first experimental measurement of the driving wavelength scaling of the attochirp using intense midinfrared sources [16-18]. The phase of the high harmonic light has been measured in gas jets of argon and xenon at three different driving wavelengths $(0.8,1.3$, and $2 \mu \mathrm{m})$ and found to agree with the expected behavior. We demonstrate that at constant intensity, a smaller attochirp is achieved in argon at $2 \mu \mathrm{m}$ compared with $0.8 \mu \mathrm{m}$. The measurements are performed by adapting the all-optical method introduced by Dudovich et al. [19]. In this method, the high harmonics dispersed through a spectrometer are generated by a two-color combined field composed of the fundamental driving field and its second harmonic. Since the total resultant electric field is no longer symmetric from one half optical cycle to the next, the centrosymmetry of the generation process is lost, resulting in both even- and odd-order harmonics. The amplitude of the even-order harmonics depends on the relative phase of the two-color combined field. By controlling the subcycle delay between the fundamental and second harmonic pulses and recording the resulting oscillations, it is possible using the semiclassical model to retrieve the emission times of the even-order harmonics, and by interpolation, that of the odd orders. In the limit of a sufficiently weak second harmonic beam the emission times correspond to the unperturbed values. It should be stressed that this method yields the in situ attochirp, independent of possible subsequent compensation, and therefore simplifies the comparison with theory.

The experimental setup is similar to the original measurement conducted by Dudovich, and is shown in Fig. 2. The laser source used in these studies is described elsewhere [16]. A $300 \mu \mathrm{m}$ thick BBO crystal, tuned out of the phase-matching angle, generates the required small

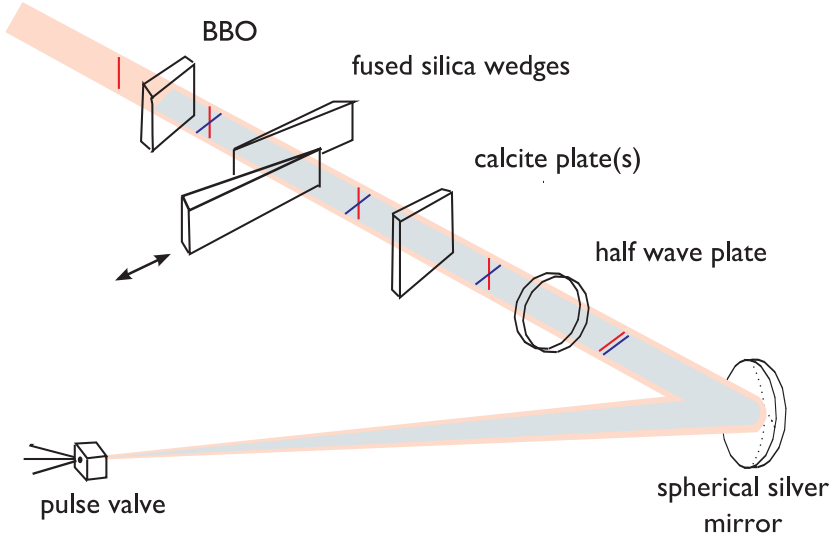

FIG. 2 (color online). Experimental setup. The two beams polarization directions are represented. 
amount of second harmonic for all three wavelengths. The group delay accumulated through propagation in air and glass must be compensated for the pulses to temporally overlap on target. This is achieved by using a number of birefringent plates (calcite) and a pair of fused silica wedges which are also used to control the fine subcycle delay between the electric fields of the two pulses. A zeroorder, half-wave plate is finally used to set the polarizations of the two pulses parallel. A silver-coated mirror of $450 \mathrm{~mm}$ focal length focuses both beams on the harmonic generation atomic jet. This source consists in a pulsed gas valve based on a piezoelectric disk and operating at $1 \mathrm{kHz}$. Backed with a pressure of $2.5 \mathrm{~atm}$, the valve opens for $100 \mu$ s into a $2 \mathrm{~mm}$ long, $1 \mathrm{~mm}$ diameter channel which allows for high densities in the generating medium, with both argon and xenon. The generated harmonics are then imaged on an XUV CCD (Andor) situated at the exit of our XUV spectrometer (Hettrick Scientific).

In order to extract the attochirp, it is necessary to select one class of trajectories. In this study, the short quantum paths are selected by the combined effects of phase matching (focus in front of the jet) and spatial filtering of the on-axis radiation by the small acceptance angle of the spectrometer.

Two typical high harmonic spectra are presented in Fig. 3(b) for xenon excited by a $21 \mathrm{TW} / \mathrm{cm}^{2}, 2 \mu \mathrm{m}$ pulse. The spectra recorded for two different $\omega-2 \omega$ total fields illustrate the change in amplitude of the even-order harmonics with phase. By contrast, the odd-order harmonics are not modulated. A pair of fused silica wedges provides attosecond control of the relative phase between the $\omega-$ $2 \omega$ fields. Figure 3(a) is a global plot of the harmonic

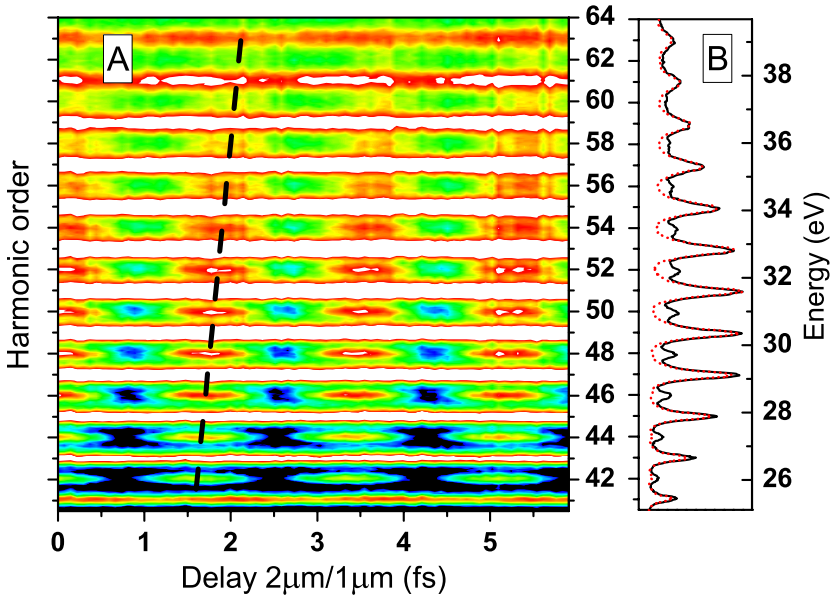

FIG. 3 (color online). (a) Full scan of $\omega-2 \omega$ delay obtained in xenon at $2 \mu \mathrm{m}$, and an intensity of $21 \mathrm{TW} / \mathrm{cm}^{2}$. The evenorder harmonics oscillate at 4 times the fundamental frequency. The dotted line serves as a guide for visualizing the shift in the delay that maximizes each even-order harmonic. (b) Two individual spectra [line outs of (a)] for specific delays maximizing (solid line) or minimizing (dashed line) the even-order harmonics. spectra as a function of this relative phase and shows both the periodic oscillations and the shift in the delay that maximizes different even-orders.

The phase between the fundamental and the second harmonic fields that maximizes each even-order harmonic (hereafter noted $\phi_{\max }$ ) is extracted from the data in Fig. 3. Following the procedure in [19], $\phi_{\max }$ is calculated using the semiclassical model and fitted to the measured values by adjusting an additive constant (the unknown offset of the relative phase between the $\omega-2 \omega$ fields). The analysis confines this treatment to only the plateau harmonics. Finally, the extraction of the attochirp relies on the correspondence between $\phi_{\max }$ and the emission times given by the model.

Figure 4 presents the attochirp for the $2 \mu \mathrm{m}$ xenon harmonics generated in the conditions of Fig. 3, and additional results obtained in argon excited by $38 \mathrm{TW} / \mathrm{cm}^{2}$, $1.3 \mu \mathrm{m}$ pulses. In energy and time units scaled to $U_{p}$ and the fundamental period, respectively, the attochirp is expected to be globally invariant. We therefore plot in those units the harmonic emission times as a function of the kinetic energy of the returning electrons. The agreement with theory (solid line) is good for the $1.3 \mu \mathrm{m}$ data (squares) and even better for the $2 \mu \mathrm{m}$ (circles) results. In absolute units, the value of the attochirp is 60 and $71 \mathrm{as} / \mathrm{eV}$ for 1.3 and $2 \mu \mathrm{m}$, respectively. These values also reflect the contribution of the different intensities of the two measurements. In order to display the pure wavelength scaling, Fig. 5 shows the product of the attochirp and the intensity as a function of the fundamental driving wavelength. The figure also includes measurements in argon for $80 \mathrm{TW} / \mathrm{cm}^{2}, 0.8 \mu \mathrm{m}$, and $71 \mathrm{TW} / \mathrm{cm}^{2}, 2 \mu \mathrm{m}$ pulses. The expected $1 / \lambda$ dependence is plotted as a solid line. The experiment clearly demonstrates the reduction with fundamental wavelength. The value of the attochirp is reduced by using a longer wavelength driver from a value

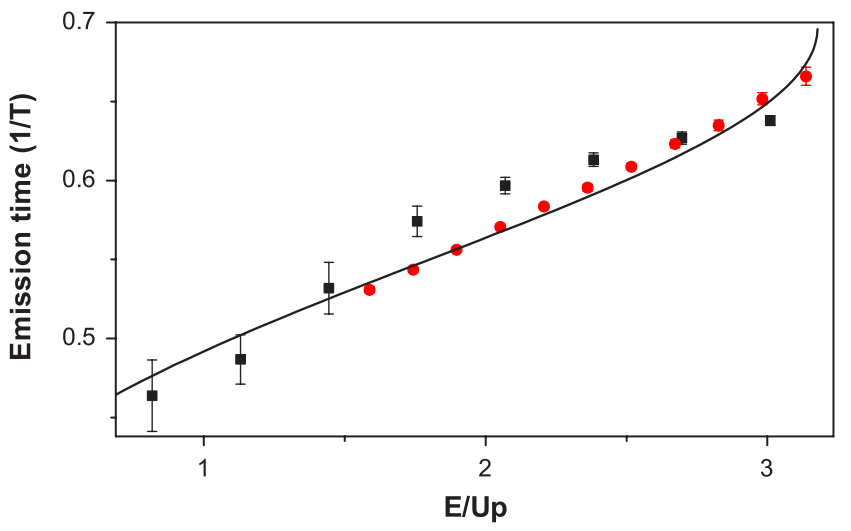

FIG. 4 (color online). Retrieved scaled emission times as a function of the scaled kinetic energy of the returning electron responsible for harmonic generation. The data from argon at $1.3 \mu \mathrm{m}$ (solid squares) and xenon at $2 \mu \mathrm{m}$ (solid circles) are compared to the semiclassical calculation (solid line). 


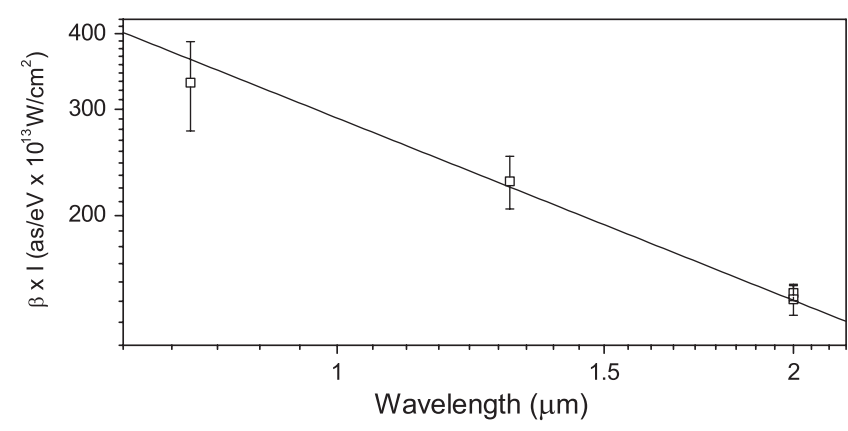

FIG. 5. Scaling of the attochirp as a function of wavelength. The product of the attochirp $\beta$ with the intensity $I$ is plotted in order to remove the effect of the peak intensity. The straight line corresponds to the predicted scaling $1 / \lambda$.

of $41.5 \mathrm{as} / \mathrm{eV}$ at $0.8 \mu \mathrm{m}$ to $21.5 \mathrm{as} / \mathrm{eV}$ at $2 \mu \mathrm{m}$. Obviously, the generated spectra were very different, with a strong extension of the cutoff at $2 \mu \mathrm{m}$. Those values consider the intrinsic attochirp of the generation process only, independently of any subsequent compensation. In those conditions, the optimum pulse duration goes down from 250 as at $0.8 \mu \mathrm{m}$ to 180 as at $2 \mu \mathrm{m}$. This corresponds to an optimal bandwidth of $\approx 12.5 \mathrm{eV}$ at $0.8 \mu \mathrm{m}$ and $\approx 20 \mathrm{eV}$ at $2 \mu \mathrm{m}$. In theory, the central frequency can be set anywhere in the generated spectra, up to $25 \mathrm{eV}$ at $0.8 \mu \mathrm{m}$ and $90 \mathrm{eV}$ at $2 \mu \mathrm{m}$.

In summary, we presented the first measurement of the attochirp of high harmonics generated with different fundamental laser wavelengths by using the two-color in situ method [19]. Our study confirms the $1 / \lambda$ scaling of the attochirp predicted by the semiclassical model. The reduction of the attochirp with wavelength at constant peak intensity leads to shorter attosecond pulses.

It may be possible in the near future to generate high harmonics at even longer wavelength. For instance, our $3.6 \mu \mathrm{m}$ source [7] at $100 \mathrm{TW} / \mathrm{cm}^{2}$ will produce a $400 \mathrm{eV}$ cutoff energy, an intrinsic chirp of $\approx 8$ as/eV and an optimum pulse duration of 100 as (without compensation). The present measurement substantiates that longer wavelengths are a viable route towards $\mathrm{x}$-ray pulses with durations approaching the atomic unit of time.
This work was performed with support from USDOE/ BES under Contracts No. DE-FG02-04ER15614 and No. DE-FG02-06ER15833. L. F. D. acknowledges support from the Hagenlocker chair. We are grateful to Dr. Linda Young (Argonne National Laboratory) for the loan of the Hettrick spectrometer.

*gdoumy@mps.ohio-state.edu

[1] P. Agostini and L.F. DiMauro, Rep. Prog. Phys. 67, 813 (2004).

[2] Y. Mairesse et al., Science 302, 1540 (2003).

[3] E. Goulielmakis et al., Science 320, 1614 (2008).

[4] G. Sansone et al., Science 314, 443 (2006).

[5] P. B. Corkum and F. Krausz, Nature Phys. 3, 381 (2007).

[6] B. Shan and Z. Chang, Phys. Rev. A 65, 011804(R) (2001).

[7] P. Colosimo et al., Nature Phys. 4, 386 (2008).

[8] K. J. Schafer, B. Yang, L. F. DiMauro, and K. C. Kulander, Phys. Rev. Lett. 70, 1599 (1993).

[9] P. B. Corkum, Phys. Rev. Lett. 71, 1994 (1993).

[10] M. Lewenstein, P. Balcou, M. Y. Ivanov, A. L'Huillier, and P. B. Corkum, Phys. Rev. A 49, 2117 (1994).

[11] J.-C. Diels and W. Rudolph, Ultrashort Laser Pulse Phenomena (Academic Press, New York, 2006), 2nd ed.

[12] J. Tate, T. Auguste, H. G. Muller, P. Salieres, P. Agostini, and L. F. DiMauro, Phys. Rev. Lett. 98, 013901 (2007).

[13] R. Lopez-Martens et al., Phys. Rev. Lett. 94, 033001 (2005).

[14] E. Gustafsson, T. Ruchon, M. Swoboda, T. Remetter, E. Pourtal, R. Lopez-Martens, P. Balcou, and A. L'Huillier, Opt. Lett. 32, 1353 (2007).

[15] S. Kazamias and P. Balcou, Phys. Rev. A 69, 063416 (2004).

[16] C. P. Hauri et al., Opt. Lett. 32, 868 (2007).

[17] T. Popmintchev, M.-C. Chen, O. Cohen, M. E. Grisham, J. J. Rocca, M. M. Murnane, and H. C. Kapteyn, Opt. Lett. 33, 2128 (2008).

[18] E. J. Takahashi, T. Kanai, Y. Nabekawa, and K. Midorikawa, Appl. Phys. Lett. 93, 041111 (2008).

[19] N. Dudovich, O. Smirnova, J. Levesque, Y. Mairesse, M. Y. Ivanov, D. M. Villeneuve, and P. B. Corkum, Nature Phys. 2, 781 (2006). 